\title{
Intensive Antihypertensive Treatment with Angiotensin Receptor Blocker Combined with Hydrocholorthiazide Reduces Urinary Angiotensinogen in Patients with Type 2 Diabetes Mellitus"
}

\author{
Satoru Kuriyama, Naoki Sugano, Kohki Takane, Akimitsu Kobayashi, Yasushi Otsuka, \\ Tatsuo Hosoya \\ Division of Kidney \& Hypertension, Department of Internal Medicine, School of Medicine, Jikei University, Tokyo, Japan \\ Email: kuriyamas218@yahoo.ac.jp
}

Received March 25, 2013; revised May 5, 2013; accepted May 18, 2013

Copyright (C) 2013 Satoru Kuriyama et al. This is an open access article distributed under the Creative Commons Attribution License, which permits unrestricted use, distribution, and reproduction in any medium, provided the original work is properly cited.

\begin{abstract}
Purpose: Local activation of rennin-angiotensin system (RAS) is involved in the progression of chronic kidney disease (CKD). One of the RAS components, angiotensinogen (AGT) has been known to be a potential surrogate biomarker for the renal RAS activity. Measuring the daily urinary excretion of AGT (U-AGT), the present study addressed whether the intensive blood pressure (BP) lowering with combined antihypertensive agents could improve such an abnormality in diabetic CKD patients. Methods: Uncontrolled hypertensive patients with type 2 diabetes with mild to moderate nephropathy previously receiving angiotensin receptor blockers (ARB) in an optimal dose alone were recruited for a better blood pressure (BP) control. Urinary specimens were subjected to a quantitative measurement of a daily urinary protein (U-prot) and U-AGT. After the baseline measurement, intensive antihypertensive therapy was attempted by switching the ARB dose to a fixed combination formula of candesartan $8 \mathrm{mg}$ plus hydrochlorthiazide (HCTZ) $6.25 \mathrm{mg}$ and the patients were followed up for 24 weeks. Comparison of parameters was then made between the values at the baseline and the end of the study. Results: At baseline, there was a significant positive correlation between U-AGT and U-prot, and between U-AGT and serum creatinine (Cr) concentration. In addition, U-AGT was inversely correlated with estimated glomerular filtration rate (e-GFR). Switching the antihypertensive regime from ARB alone to the combined ARB/HCTZ significantly reduced BP, U-AGT and U-prot. The magnitude of the reduction in U-prot was positively correlated with that in U-AGT. A stepwise regression analysis showed that HbA1c, e-GFR and the reduction in U-prot in response to the intensive antihypertensive therapy were positively correlated with the reduction in U-AGT. Conclusion: U-AGT is increased and positively correlated with U-prot in patients with type 2 diabetic nephropathy. Intensive antihypertensive treatment with ARB combined with HCTZ reduces both U-AGT and U-prot, presumably via an amelioration of an accelerated renal RAS activity. These data also suggest that U-AGT can be used as a potential therapeutic surrogate biomarker for the activated renal RAS in patients with diabetic nephropathy.
\end{abstract}

Keywords: Hypertension; Angiotensinogen; Proteinuria; Renin-Angiotensin System; ARB; Diabetic Nephropathy

\section{Introduction}

The role of the rennin-angiotensin system (RAS) in the pathophysiology of chronic renal disease (CKD) has been highlighted in relation to the local RAS activity in the kidney. Experimental studies revealed that intrarenal angiotensin II (AII) is regulated by a mechanism independent of circulating AII, and that elevated levels of intrarenal AII may link to renal functional derangement

${ }^{*}$ Conflict of interest: No conflict of interest is declared. and tissue injury, leading to the progression of CKD [1,2]. Of importance is that the source of renal AII is not only derived from the systemic circulation but also from the locally formed AII substrate, angiotensinogen (AGT) [3-8]. Animal studies suggest that AGT levels in the renal tissues reflect the activity of intrarenal RAS [1-6,8]. In human, Yamamoto et al. showed that urinary AGT levels were elevated in patients with CKD [9]. In addition, similar results have recently demonstrated that urinary AGT levels in renal tissues are increased in human 
IgA glomerulonephritis [10,11]. These studies indicated that urinary AGT is mainly derived from the renal tissues, and that it can be used as a potential surrogate biomarker for the local RAS activity in CKD.

A plethora of reports have proven that the effective antihypertensive interventions are undoubtedly associated with a decline in urinary protein excretion, contributing to renoprotection. However, it is also obvious that treatment with RAS inhibitor, either ACEIs or ARBs, is successful in mitigating CKD in a BP independent manner [12-14]. However, reports on whether BP lowering unequivocally induces reduction in urinary AGT are still scant. Reviewing the literatures, Bakris et al. have shown that in order to achieve lower BP of less than 130/80 $\mathrm{mmHg}$, more than two drugs are needed in most patients [15]. Indeed, many guidelines for the management of hypertension have recommended that combination of multiple antihypertensive agents with different pharmacological mode of action is more efficacious than a single agent alone in terms of renal protection [16]. The combination of an AII receptor blocker (ARB) and hydrochlorothiazide (HCTZ) has been widely recognized as a preferable prescription, because combining ARB with HCTZ exerts a complementary antihypertensive effect by suppressing RAS with the former and body fluid system with the latter, which provides a greater reduction in BP than either agent alone. A fixed combination formulation of ARB plus HCTZ may be more advantageous because of a better adherence to therapy and less side effects [17].

With the above-mentioned background taken into consideration, the present study made an attempt to investigate 1) whether abnormality exists in urinary AGT excretion in patients with type 2 diabetic nephropathy, and 2) whether the intensive antihypertensive treatment consisting of ARB plus HCTZ can improve such abnormality.

\section{Patients \& Methods}

\subsection{Patients}

Enrolled in the present study were patients with type 2 diabetic nephropathy (DN) (average age: $66.4+/-9.7$, male/female: 20/15) who agreed to participate in the collection of 24 hour urine collection for the evaluation of a daily urinary protein (U-prot) and angiotensinogen excretion (U-AGT). Patients whoes semi-quantitative measurement of urinary test was positive for proteinuria at least once were included in the study. The basic clinical characteristics of the patients enrolled are shown in Table 1 . BP at the entry was $148+/-9 \mathrm{mmHg}$ in systolic and $88+/-5 \mathrm{mmHg}$ in diastolic. The diagnosis of diabetes mellitus was made based upon clinical and laboratory findings. Patients all satisfied the American Diabetes Association (ADA) diagnostic criteria of diabetes mellitus [18].
Table 1. Patient characteristics.

\begin{tabular}{cc}
\hline Age $(\mathrm{y})$ & $66.4 \pm 9.7$ \\
Male/Female & $\mathrm{M} / \mathrm{F}=20 / 15$ \\
$\mathrm{BMI}\left(\mathrm{kg} / \mathrm{m}^{2}\right)$ & $23.8 \pm 2.8$ \\
$\mathrm{SBP} / \mathrm{DBP}(\mathrm{mmHg})$ & $148 \pm 9 / 88 \pm 5$ \\
Serum Cr $(\mathrm{mg} / \mathrm{dL})$ & $2.3 \pm 1.5$ \\
$\mathrm{Hb}(\mathrm{mg} / \mathrm{dL})$ & $12.2 \pm 1.8$ \\
$\mathrm{HbAlc}(\%)$ & $6.1 \pm 1.2$ \\
$\mathrm{~K}(\mathrm{mEq} / \mathrm{l})$ & $4.6 \pm 0.4$ \\
$\mathrm{Ccr}\left(\mathrm{ml} / \mathrm{min} / 1.73 \mathrm{~m}^{2}\right)$ & $50.6 \pm 37.4$ \\
$\mathrm{e}-\mathrm{GFR}\left(\mathrm{ml} / \mathrm{min} / 1.73 \mathrm{~m}^{2}\right)$ & $33.0 \pm 20.9$ \\
$\mathrm{U}-\mathrm{prot}(\mathrm{g} / \mathrm{day})$ & $1.5 \pm 1.3$ \\
$\mathrm{U}-\mathrm{AGT}(\mu \mathrm{gg} / \mathrm{g} \cdot \mathrm{Cr})$ & $331 \pm 351$ \\
$\mathrm{U}-\mathrm{AGT}(\mu \mathrm{gg} / \mathrm{day})$ & $386 \pm 389$ \\
$\mathrm{ARB}$ use $(\%)$ & 100 \\
Insulin use $(\%)$ & 63 \\
\hline
\end{tabular}

\subsection{Treatment Plans}

The inclusion criteria were outpatients whose BP was more than $130 / 80 \mathrm{mmHg}$ despite previous antihypertensive agents (ARB alone) prescribed for more than 3 months prior to the study entry. Such ARBs had been prescribed in the optimal dose of each including candesartan $8 \mathrm{mg}$ in 12 cases, losartan $50 \mathrm{mg}$ in 8 , ormesartan $40 \mathrm{mg}$ in 8 , valsartan $80 \mathrm{mg}$ in 7 cases. At the entry, ARBs were switched to a single formulation tablet of candesratan $8 \mathrm{mg}$ plus hydrochrolothiazide (HCTZ) 6.25 $\mathrm{mg}\left(\right.$ Ecard $\left.\mathrm{HD}^{\mathbb{R}}\right)$. These strategies were based upon an assumption that the combination therapies might exert more intense reduction in U-prot and U-AGT than ARB alone. Addition of HCTZ to ARB has been widely recommended to effectively reduce U-prot. Due to an ethical reason, drugs which are known to affect renal outcomes such as statins, anti-platelet agents, erythrocyte stimulating agents, remained unchanged throughout the study period. Dietary interventions were also kept unchanged.

The institution received prior ethics committee and or institutional review board approval (\# 193), and the trial was conducted in accordance with the principles of Good Clinical Practice and the ethical principles of the concurrent Declaration of Helsinki which also protected the privacy of the patients. All patients gave written informed consent before study enrollment. Clinical trial Number by UMIN was 000001950 . 


\subsection{Measurement of Urinary Parameters}

Blood samples were obtained between 10 - 12 AM at the sitting position after more than 30 minutes rest. One day before the blood tests, 24 hours urine collection was made and samples were subjected to the quantitative measurement of a daily excretion on urinary protein and AGT. AGT was measured by a sandwich EIA method based on the theory reported elsewhere (19). The measuring plate is commercially available as a Human Total Angiotensinogen Assay Kit-IBL (Takasaki, Japan). The detection limit of this assay is estimated to be $0.30 \mathrm{ng} / \mathrm{ml}$.

\subsection{Statistical Analyses}

Student t-test, the Chi-square test, and the Stepwise regression analysis were carried out with JMP 9.0 software. The computer used for the analysis was a Dynabook Satellite 2590X (Toshiba, Tokyo, Japan). Because of their skewed distribution, logarithmic transformation of AGT values was performed as the geometric means with $95 \%$ confidence intervals in some parts of the statistical analyses. Data were presented as mean $+/-\mathrm{SD}$, unless otherwise indicated. A p-value of less than 0.05 was considered statistically significant.

\section{Results}

Figure 1 shows the effect of switching the previous treatment with ARB alone to the intensive therapy with ARB plus HCTZ on BP, U-prot (a total daily urinary excretion) and U-AGT (a total daily urinary excretion of AGT). After introducing the intensive antihypertensive therapy (so-called "treatment"), both systolic (from 148 $+/-9$ to $132+/-5 \mathrm{mmHg}, \mathrm{p}<0.001)$ and diastolic BP (from $88+/-5$ to $82+/-4 \mathrm{mmHg}, \mathrm{p}<0.001$ ) were reduced significantly. Similarly, both U-prot (from $1.5+/-$ 1.3 to $1.2+/-0.8 \mathrm{~g} /$ day, $\mathrm{p}<0.001$ ) and U-AGT (from $386+/-389$ to $208+/-204 \mu \mathrm{g} /$ day, $\mathrm{p}<0.001$ ) were reduced in the majority of patients in the 24 weeks treatment period.

Figure 2 shows the relationship between pre-treatment U-AGT and U-prot. There was a close correlation between the two parameters (Figure 1, right). When logarithmic transformation was considered, such a correlation appeared to be more apparent (Figure 1, left).

Figure 3 show the relationship between pre-treatment AGT and serum Cr concentration or e-GFR. There was a significant positive correlation between serum $\mathrm{Cr}$ concentration and pre-treatment U-AGT, and in exchange negative correlation between serum $\mathrm{Cr}$ concentration and e-GFR.

Figure 4 shows relationship between the reduction ratio of a daily urinary protein excretion $(\Delta U$-prot) and that of a daily urinary excretion of AGT ( $\Delta U-A G T)$, in response the intensive antihypertensive treatment. The former was significantly positively correlated with the latter.

Figure 5 shows relationship between the total amount of the reduced daily urinary excretion (U-prot, plotted in the $\mathrm{X}$-axis) and the ratio of the reduction in a daily urinary excretion of AGT ( $\Delta \mathrm{U}-\mathrm{AGT}$, plotted in the Y-axis). Similar to Figure 2, the former was significantly positively correlated with the latter.
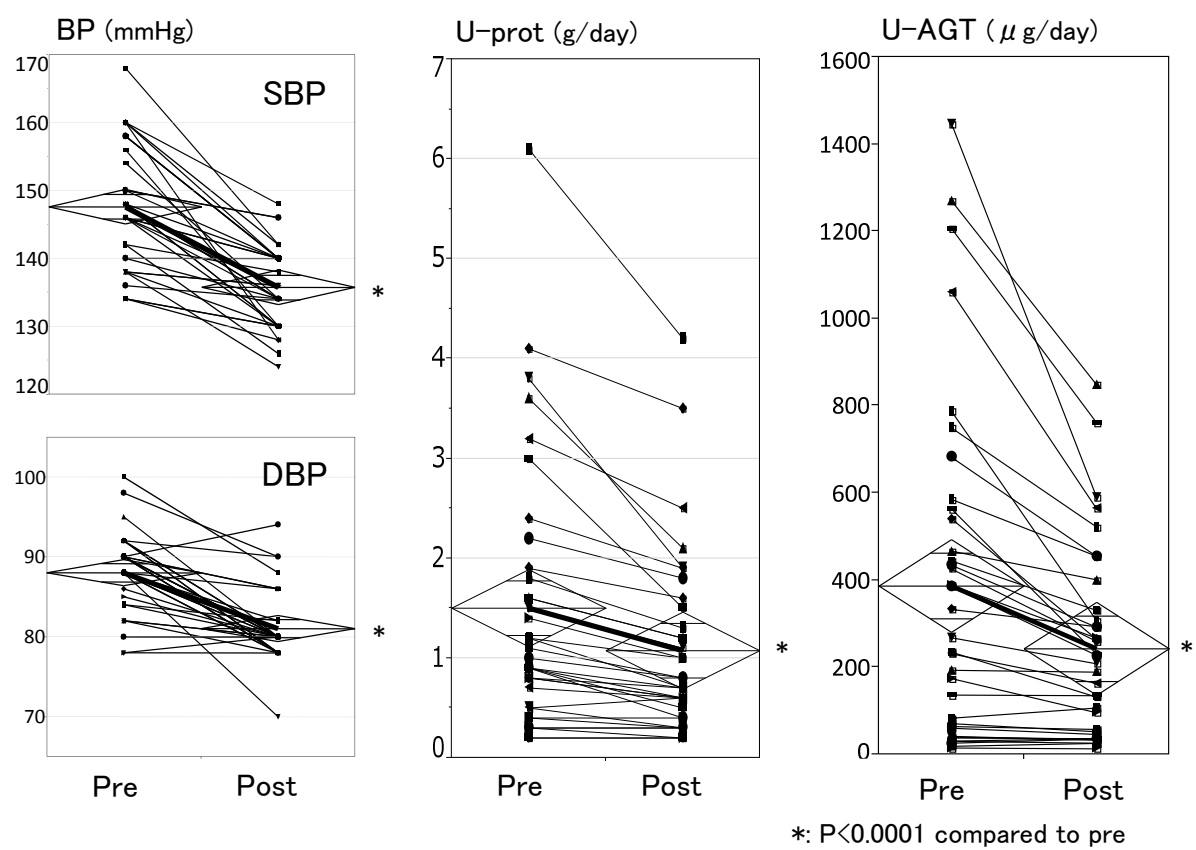

Figure 1. Effect of intensive antihypertensive therapy with ARB/HCTZ. 

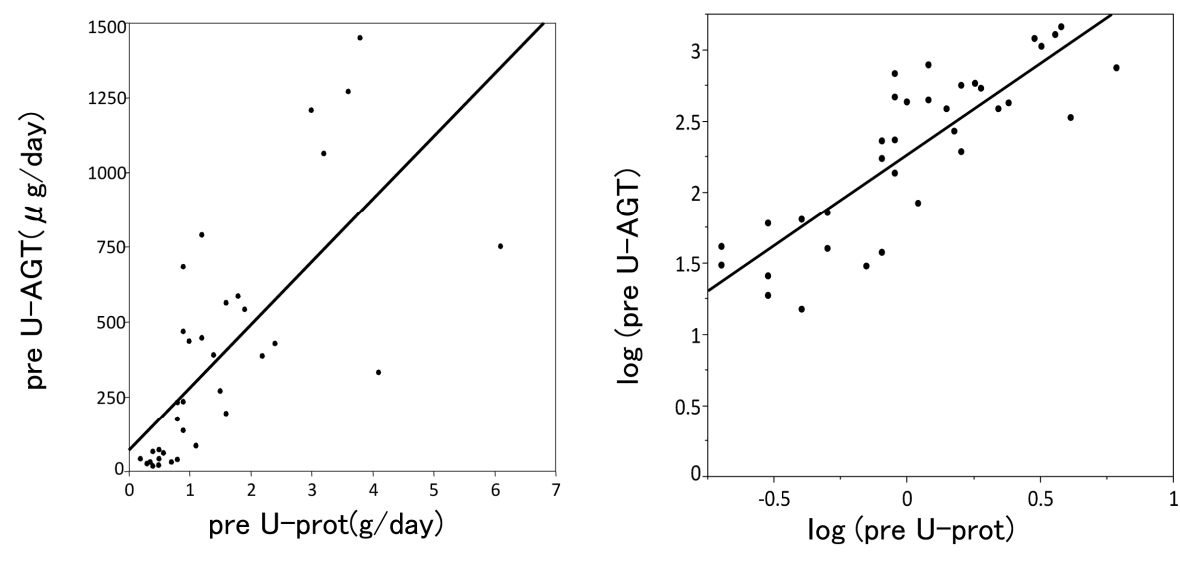

$y=210 x+71.5, r=0.72, P<0.0001$ $y=1.28 x+2.27, r=0.84, P<0.0001$

Figure 2. Relationship between preU-AGT and preU-prot.
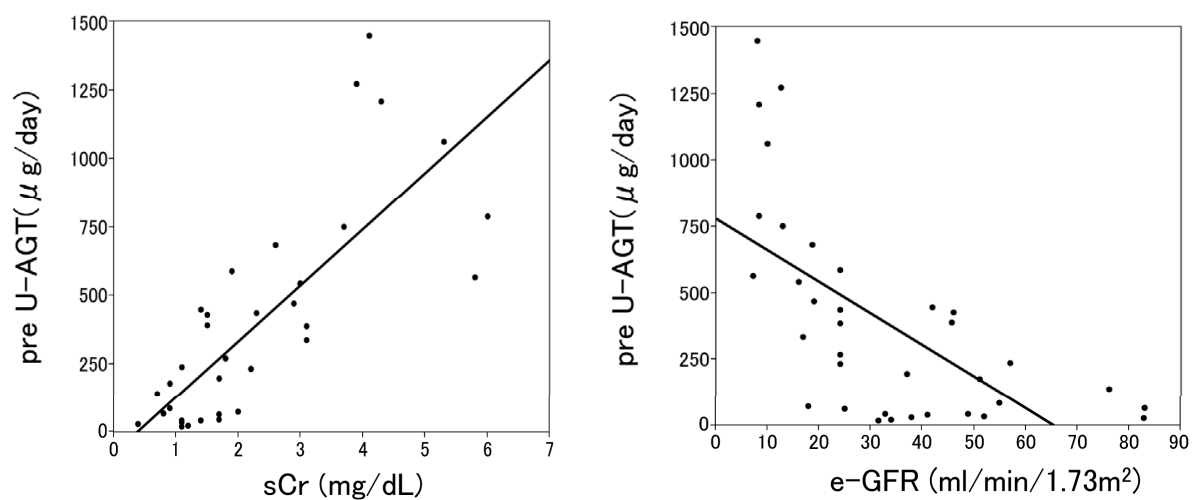

$y=206 x-79.3, r=0.78, P<0.0001$

$y=-11.9 x+779, r=-0.63, P<0.0001$

Figure 3. Relationship between preU-AGT and Cr, and preU-AGT and e-GFR.

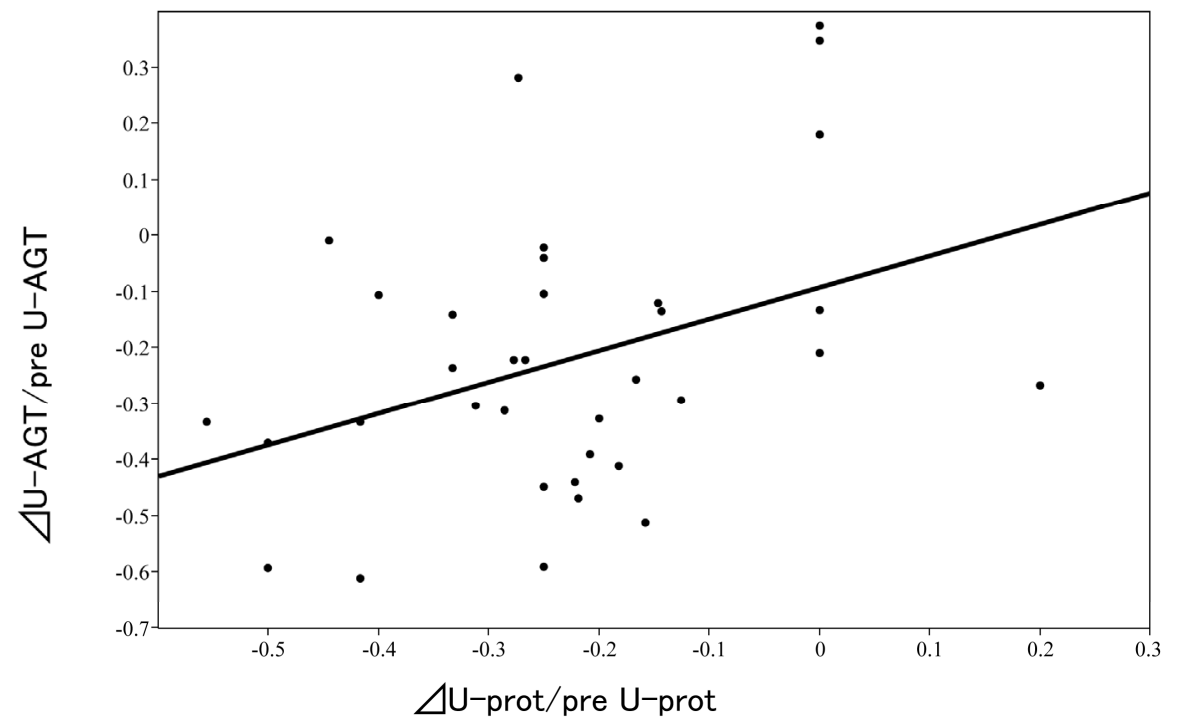

$y=0.561 x-0.092, r=0.3733, P=0.027$

Figure 4. Relationship between $\Delta \mathrm{U}$-AGT ratio and $\Delta \mathrm{U}$-prot ratio. 


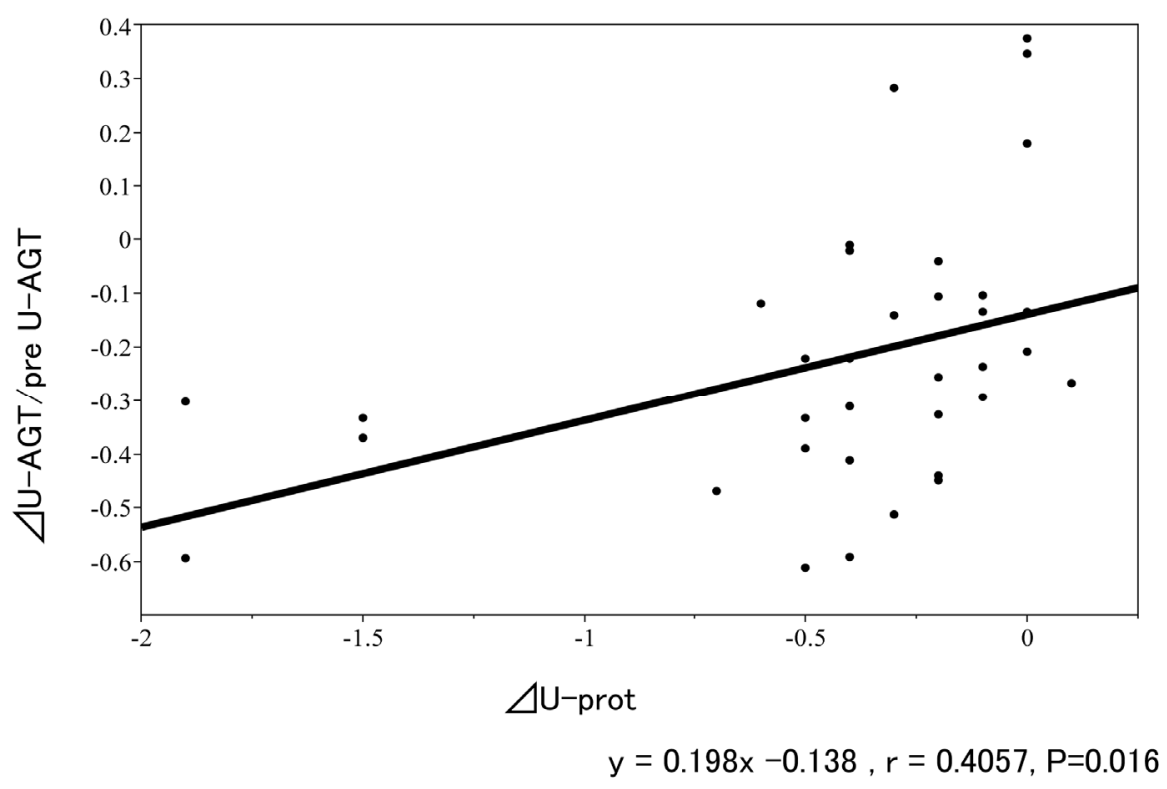

Figure 5. Relationship between $\Delta \mathrm{U}$-AGT ratio and $\Delta \mathrm{U}$-prot.

Table 2 shows the result of a stepwise regression analysis to explain pre-treatment value of U-AGT. The factors affecting such values were predicted to be pre-treatment U-prot, HbA1c and e-GFR.

Table 3 shows the result of a stepwise regression analysis to explain the reduction in U-AGT. Such predictors include the reduction in U-prot, e-GFR and HbAlc.

\section{Discussion}

This clinical study presented two major findings. First, U-AGT is substantially increased and positively correlated with U-prot in patients with mild to moderate type 2 diabetic nephropathy, suggesting that this biomarker

Table 2. A stepwise regression analysis to explain pre $U$ AGT.

\begin{tabular}{cccc}
\hline Parameters & $\beta$ value & $\mathrm{F}$ & p-value \\
\hline U-prot (pre) & 151.5 & 24.3 & $2.86 \mathrm{e}^{-5}$ \\
HbA1c & 91.55 & 9.6 & 0.00419 \\
e-GFR & -5.86 & 8.7 & 0.00601 \\
DBP & 13.9 & 3.9 & 0.0574 \\
\hline
\end{tabular}

Table 3. A stepwise regression analysis to explain $\Delta \mathrm{U}$-AGT.

\begin{tabular}{cccc}
\hline Parameters & $\beta$ value & $\mathrm{F}$ & $\mathrm{p}$-value $(\mathrm{p}>\mathrm{F})$ \\
\hline$\Delta$ U-prot & 205.6 & 20.5 & $8.15 \mathrm{e}^{-5}$ \\
e-GFR & 2.84 & 7.2 & 0.01161 \\
HbA1c & -37.54 & 5.2 & 0.03011 \\
\hline
\end{tabular}

can be used as a potential surrogate marker for the renal RAS. Second, intensive antihypertensive treatment with ARB plus HCTZ reduces both U-AGT and U-ptot, suggesting that intensive BP lowering may ameliorate an accelerated activity of renal RAS.

\subsection{Urinary AGT as a Biomarker for Renal RAS}

Notwithstanding that a part of the activity of systemic RAS can be clinically assessed as plasma renin activity (PRA), up until several years ago there was no accurate surrogate biomarker to estimate the activity of the local RAS in the kidney. Assessment of intrarenal RAS status is essential to understand the mechanisms that mediate the pathophysiology of renal function and injury [1,2]. Recent preclinical evidence suggests that intrarenal RAS activity is regulated by changes in local AGT levels [1-4] and that urinary excretion of AGT reflects intrarenal AGT production [3-8]. Following the substantial amount of animal experiments showing that U-AGT can be a new marker of intrarenal RAS status, similar results on the clinical significance of U-AGT have emerged from human studies in patients with CKD including type 1 diabetics with early stage nephropathy and primary glomerulonephritis [9-11,19-21]. Considering that all of those human studies were performed measuring U-AGT levels in a semi-quantitative way, not quantifying the total amount of daily excretion of AGT, the present study carried out quantitative measurement of a 24-hour daily protein excretion and AGT for the purpose of evaluating U-AGT status with more accuracy.

Inasmuch as the present finding does not address "cause \& effect" scenario, a close association of U-AGT 
with U-prot confirms that U-AGT can be used as a biomarker for the increase renal RAS activity. Saito et al. demonstrated that urinary AGT levels are increased in type 1diabetic subjects and that the early dissociation between urinary albumin levels and AGT levels may imply increased urinary AGT levels precede increased urinary albumin levels. They proposed that urinary AGT levels may serve as a sensitive early marker for intrarenal RAS activity and can be one of the earliest predictors of diabetic nephropathy in diabetic patients [19]. Similarly, in type 2 diabetic patients, increased urinary levels of AGT have accumulated $[20,21]$. The mechanisms by which the up-regulated renal AGT has a deleterious effect on renal tissue can be accounted for by the high glucose milieu in diabetic nephropathy, leading to an overproduction of local AII [22].

As to the urinary protein excretion which sometimes reaches the range of nephrotic syndrome in overt diabetic nephropathy, concerns remain about the urinary leakage of AGT from the systemic circulation. However, this is very unlikely because a large molecular weight (MW) substance like AGT, a precursor of AII, (MW: 50 - 60 $\mathrm{kDa}$ ) in the systemic circulation hardly penetrates basal membrane of glomeruli of the kidney. Indeed, to determine if circulating AGT is a source of urinary AGT, Kobori et al. infused human AGT into hypertensive and normotensive rats [6]. However, human AGT was detectable in plasma but not detectable in the urine of rats, indicating quite limited glomerular permeability and/or tubular degradation. In addition, Katsurada et al. found that there was a strong correlation between urinary AGT levels and urinary protein levels, but no link was found between plasma AGT and urinary protein levels [23].

In non-diabetic population, Nishiyama et al. reported that urinary AGT levels were higher in IgA nephropathy patients than in healthy volunteers and that treatment with an ARB reduced AGT levels, suggesting the significance of urinary AGT as a potential biomarker for local RAS in the kidney [24]. Jang et al. also reported that urinary AGT level reflects intrarenal AGT expression and correlates with the extent of proteinuria and renal function, indicating the intrarenal compartment as the main source of urinary AGT in proteinuric IgA nephropathy patients [25]. Recently, Kim et al. also support that urinary AGT is a biomarker in patients with IgA nephropathy [26]. These findings strongly endorse the hypothesis that urinary AGT is derived from the AGT produced and secreted by renal tissue, probably the proximal tubules, and is undoubtedly not from plasma. These observations are in good accordance with our present findings and reinforce the notion that urinary AGT is a potential novel biomarker of the intrarenal RAS in CKD patients.

\subsection{Effect of Intensive Antihypertensive Treatment on Urinary AGT}

Many reports have shown that effective antihypertensive interventions are associated with a decline in U-prot, and contribute to renoprotection [27-31]. However, it is also obvious that treatment with RAS inhibitor, either ACEIs or ARBs, is successful in mitigating CKD in a BP independent manner [10-12]. Although clinical studies to observe the effects of ARB on urinary AGT are scarce and a matter of controversy, it appears that ARB reduces urinary AGT in most of the studies [19-21,24]. There was one controversial report that did not show any association between urinary AGT levels and BP lowering with ARB or ACE-I [32].

The majority of guidelines for the management of hypertension have recommended that combination of multiple antihypertensive agents with different pharmacological mode of action is more efficacious than a single agent alone in terms of renal protection [16,33,34]. Combining ARB with HCTZ exerts a complementary pharmacological effect by suppressing RAS with the former and body fluid system with the latter. A fixed combination formulation of ARB plus HCTZ may be more advantageous because of a better adherence to therapy. Hosoya et al. have reported that after switching from the preceding antihypertensive treatment with ARB alone a fixed combination formula tablet of losartan and HCTZ efficiently reduced $\mathrm{BP}$, albumin-creatinine ratio (ACR) and B-type natriuretic peptide (BNP), suggesting that intensive therapy with ARB plus HCTZ has a beneficial effect not only on kidney function but also on cardiac protection [17]. Using the similar clinical interventional method, the present study clarified that the switching therapy from ARB alone to a fixed combination formula of ARB plus HCTZ is effective in reducing in both U-AGT and U-prot.

Whether urinary AGT is a superior biomarker to the recently-reported other urinary RAS components (AII, aldosterone and renin) is still a matter for debate, and future studies will disclose which one would be an appropriate marker to more accurately estimate local RAS activity.

\subsection{Limitation of the Study}

There are several limitations in this exploratory study. A larger number of patients and a longer observation period with a contol-based prospective design must be carried out in the future evaluation on the role of urinary AGT.

In summary, the present study indicates that increase in urinary AGT is correlated well with urinary protein excretion in patients with type 2 diabetic nephropathy and that intensive antihypertensive treatment ameliorates such abnormalities. These data support the notion that urinary AGT is a potential tool for the determination of 
intrarenal RAS status and is associated with renal derangement.

\section{Acknowledgement}

A part of this work was presented at the $55^{\text {th }}$ Annual Meeting of the Japanese Society of Nephrology held in Tokyo, Japan, on June $1^{\text {st }}, 2012$.

\section{REFERENCES}

[1] L. G. Navar, L. M. Harrison-Bernard, A. Nishiyama and H. Kobori, "Regulation of Intrarenal Angiotensin II in Hypertension," Hypertension, Vol. 39, 2002, pp. 316-322. doi:10.1161/hy0202.103821

[2] H. Kobori, M. Nangaku, L. G. Navar and A. Nishiyama, "The Intrarenal Renin-Angiotensin System: From Physiology to the Pathobiology of Hypertension and Kidney Disease," Pharmacological Reviews, Vol. 59, No. 3, 2007, pp. 251-287. doi:10.1124/pr.59.3.3

[3] H. Kobori, Y. Ozawa, Y. Suzaki, M. C. Prieto-Carrasquero, A. Nishiyama, T. Shoji, E. P. Cohen and L. G. Navar, "Young Scholars Award Lecture: Intratubular Angiotensinogen in Hypertension and Kidney Diseases," American Journal of Hypertension, Vol. 19, No. 5, 2006, pp. 541-550. doi:10.1016/j.amjhyper.2005.11.014

[4] H. Kobori, Y. Ozawa, R. Satou, A. Katsurada, K. Miyata, N. Ohashi, N. Hase, Y. Suzaki, C. D. Sigmund and L. G. Navar, "Kidney-Specific Enhancement of ANG II Stimulates Endogenous Intrarenal Angiotensinogen in Genetargeted Mice," American Journal of Physiology-Renal Physiology, Vol. 293, No. 3, 2007, pp. F938-F945. doi:10.1152/ajprenal.00146.2007

[5] H. Kobori, L. M. Harrison-Bernard and L. G. Navar, "Urinary Excretion of Angiotensinogen Reflects Intrarenal Angiotensinogen Production," Kidney International, Vol. 61, 2002, pp. 579-585.

doi:10.1046/j.1523-1755.2002.00155.x

[6] H. Kobori, A. Nishiyama, L. M. Harrison-Bernard and L. G. Navar, "Urinary Angiotensinogen as an Indicator of Intrarenal Angiotensin Status in Hypertension," Hypertension, Vol. 41, 2003, pp. 42-49. doi:10.1161/01.HYP.0000050102.90932.CF

[7] H. Kobori, A. B. Alper Jr., R. Shenava, A. Katsurada, T. Saito, N. Ohashi, M. Urushihara, K. Miyata, R. Satou, L. L. Hamm and L. G. Navar, "Urinary Angiotensinogen as a Novel Biomarker of the Intrarenal Renin-Angiotensin System Status in Hypertensive Patients," Hypertension, Vol. 53, 2009, pp. 344-350. doi:10.1161/HYPERTENSIONAHA.108.123802

[8] H. Kobori, Y. Ozawa, Y. Suzaki and A. Nishiyama, "Enhanced Intrarenal Angiotensinogen Contributes to Early Renal Injury in Spontaneously Hypertensive Rats," Journal of the American Society of Nephrology, Vol. 16, No. 7, 2005, pp. 2073-2080. doi:10.1681/ASN.2004080676

[9] T. Yamamoto, T. Nakagawa, H. Suzuki, N. Ohashi, H. Fukasawa, Y. Fujigaki, A. Kato, Y. Nakamura, F. Suzuki and A. Hishida, "Urinary Angiotensinogen as a Marker of
Intrarenal Angiotensin II Activity Associated with Deterioration of Renal Function in Patients with Chronic Kidney Disease," Journal of the American Society of Nephrology, Vol. 18, No. 5, 2007, pp. 1558-1565. doi:10.1681/ASN.2006060554

[10] H. Kobori, A. Katsurada, Y. Ozawa, R. Satou, K. Miyata, N. Hase, Y. Suzaki and T. Shoji, "Enhanced Intrarenal Oxidative Stress and Angiotensinogen in IgA Nephropathy Patients," Biochemical and Biophysical Research Communications, Vol. 358, No. 1, 2007, pp. 156-163. doi:10.1016/i.bbrc.2007.04.105

[11] M. Takamatsu, M. Urushihara, S. Kondo, M. Shimizu, T. Morioka, T. Oite, H. Kobori and S. Kagami, "Glomerular Angiotensinogen Protein Is Enhanced in Pediatric IgA Nephropathy," Pediatric Nephrology, Vol. 23, No. 8, 2008, pp. 1257-1267. doi:10.1007/s00467-008-0801-6

[12] D. Russo, R. Minutolo, A. Pisani, R. Esposito, G. Signoriello, M. Andreucci and M. M. Balletta, "Coadministration of Losartan and Enalapril Exerts Additive Antiproteinuric Effect in IgA Nephropathy," American Journal of Kidney Diseases, Vol. 38, No. 1, 2001, pp. 18-25. doi:10.1053/ajkd.2001.25176

[13] K. F. Hilgers and J. F. Mann, "ACE Inhibitors versus AT(1) Receptor Antagonists in Patients with Chronic Renal Disease," Journal of the American Society of Nephrology, Vol. 13, No. 4, 2002, pp. 1100-1108.

[14] R. Coppo, L. Peruzzi, A. Amore, A. Piccoli, P. Cochat, R. Stone, M. Kirschstein and T. Linné, "IgACE: A Placebo-Controlled, Randomized Trial of AngiotensinConverting Enzyme Inhibitors in Children and Young People with IgA Nephropathy and Moderate Proteinuria," Journal of the American Society of Nephrology, Vol. 18, No. 6, 2007, pp. 1880-1888.

doi:10.1681/ASN.2006040347

[15] G. L. Bakris, M. Williams, L. Dworkin, W. J. Elliott, M. Epstein, R. Toto, K. Tuttle, J. Douglas, W. Hsueh and J. Sower, "Preserving Renal Function on Adults with Hypertension ans Diabetes: A Consensus Approach. National Kidney Foundation Hypertension and Diabetes Executive Committees Working Group," American Journal of Kidney Diseases, Vol. 36, No. 3, 2000, pp. 646-661. doi:10.1053/ajkd.2000.16225

[16] B. Waeber, "Achieving Blood Pressure Targets in the Management of Hypertension," Blood Pressure, Vol. 10, No. 2, 2001, pp. 6-12. doi:10.1080/080370501750275839-1

[17] T. Hosoya, S. Kuriyama, I. Ohno, T. Kawamura, M. Ogura, M. Ikeda, M. Ishikawa, F. Hayashi, T. Kanai, H. Tomonari, M. Soejima, K. Akaba and G. Tokudome, "Antihypertensive Effect of a Fixed-Dose Combination of Losartan/Hydrochlorothiazide in Patients with Uncontrolled Hypertension: A Multicenter Study," Clinical and Experimental Nephrology, Vol. 16, No. 2, 2012, pp. 269278. doi:10.1007/s10157-011-0564-4

[18] American Diabetes Association, "Diagnosis and Classification of Diabetes Mellitus," Diabetes Care, Vol. 35, Suppl. 1, 2012, pp. s64-s71. doi:10.2337/dc12-s064

[19] T. Saito, M. Urushihara, Y. Kotani, S. Kagami and H. Kobori, "Increased Urinary Angiotensinogen Is Precedent 
to Increased Urinary Albumin in Patients with Type 1 Diasbetes," The American Journal of the Medical Sciences, Vol. 338, 2009, pp. 478-480. doi:10.1097/MAJ.0b013e3181b90c25

[20] S. Ogawa, H. Kobori, N. Ohashi, M. Urushihara, A. Nishiyama, T. Mori, T. Ishizuka, K. Nako and S. Ito, "Angiotensin II Type 1 Receptor Blockers Reduce Urinary Angiotensinogen Excretion and the Levels of Urinary Markers of Oxidative Stress and Inflammation in Patients with Type 2 Diabetic Nephropathy," Biomark Insights, Vol. 4, 2009, pp. 97-102.

[21] M. Sawaguchi, S. I. Araki, H. Kobori, M. Urushihara, M. Haneda, D. Koya, A. Kashiwagi, T. Uzu and H. Maegawa, "Association between Urinary Angiotensinogen Levels and Renal and Cardiovascular Prognoses in Patients with Type 2 Diabetes Mellitus," Journal of Diabetes Investigation, Vol. 3, No. 3, 2012, pp. 318-324.

[22] D. E. Casarini, "Upregulation of Intrarenal Angiotensinogen in Diabetes," Hypertension Research, Vol. 33, 2010, pp. 1174-1181. doi:10.1038/hr.2010.163

[23] A. Katsurada, Y. Hagiwara, K. Miyashita, R. Satou, K. Miyata, N. Ohashi, L. G. Navar and H. Kobori, "Novel Sandwich ELISA for Human Angiotensinogen," American Journal of Physiology-Renal Physiology, Vol. 293, No. 3, 2007, pp. F956-F960. doi:10.1152/ajprenal.00090.2007

[24] A. Nishiyama, Y. Konishi, N. Ohasi, T. Morikawa, M. Urushihara, I. Maeda, M. Hamada, M. Kishida, H. Hitomi, N. Shirahashi, H. Kobori and M. Imanishi, "Urinary Angiotensinogen Reflects the Activity of Intrarenal Renin Angiotensin System in Patients with IgA Nephropathy," Nephrology Dialysis Transplantation, Vol. 26, No. 1, 2011, pp. 170-177. doi:10.1093/ndt/gfq371

[25] H. R. Jang, S. M. Kim, Y. J. Lee, J. E. Lee, W. Huh, D. J. Kim, H. Y. Oh and Y. G. Kim, "The Origin and the Clinical Significance of Urinary Angiotensinogen in Proteinuric IgA Nephropathy Patients," Annals of Internal Medicine, 2011.

[26] Y. G. Kim, S. B. Song, S. H. Lee, J. Y. Moon, K. H. Jeong, T. W. Lee and C. G. Ihm, "Urinary Angiotensinogen as a Predictive Marker in Patients with Immunoglobulin A Nephropathy," Clinical and Experimental Nephrology, Vol. 15, No. 5, 2011, pp. 720-726. doi:10.1007/s10157-011-0475-4

[27] B. M. Brenner, M. E. Cooper, D. de Zeeuw, W. F. Keane, W. E. Mitch, H. H. Parving, G. Remuzzi, S. M. Snapinn, Z. Zhang and S. Shahinfar, "Effects of Losartan on Renal and Cardiovascular Outcomes in Patients with Type 2 Diabetes and Nephropathy," New England Journal of Medicine, Vol. 345, 2001, pp. 861-869. doi:10.1056/NEJMoa011161

[28] H. H. Parving, H. Lehnert, J. Bröchner-Mortensen, R. Gomis, S. Andersen and P. Arner, "Irbesartan in Patients with Type 2 Diabetes and Microalbuminuria Study Group. The Effect of Irbesartan on the Development of Diabetic Nephropathy in Patients with Type 2 Diabetes," New England Journal of Medicine, Vol. 345, 2001, pp. 870878. doi:10.1056/NEJMoa011489

[29] H. Makino, M. Haneda, T. Babazono, T. Moriya, S. Ito, Y. Iwamoto, R. Kawamori, M. Takeuchi and S. Katayama,
"Prevention of Transition from Incipient to Overt Nephropathy with Telmisartan in Patients with Type 2 Diabetes," Diabetes Care, Vol. 30, No. 6, 2007, pp. 1577-1578. doi:10.2337/dc06-1998

[30] G. Viberti and N. M. Wheeldon, "MicroAlbuminuria Reduction with VALsartan (MARVAL) Study Investigators. Microalbuminuria Reduction with Valsartan in Patients with Type 2 Diabetes Mellitus: A Blood Pressure-Independent Effect," Circulation, Vol. 106, 2002, pp. 672-678. doi:10.1161/01.CIR.0000024416.33113.0A

[31] G. Mancia, G. De Backer, A. Dominiczak, R. Cifkova, R. Fagard, G. Germano, G. Grassi, A. M. Heagerty, S. E. Kjeldsen, S. Laurent, K. Narkiewicz, L. Ruilope, A. Rynkiewicz, R. E. Schmieder, H. A. Boudier, A. Zanchetti, A. Vahanian, J. Camm, R. De Caterina, V. Dean, K. Dickstein, G. Filippatos, C. Funck-Brentano, I. Hellemans, S. D. Kristensen, K. McGregor, U. Sechtem, S. Silber, M. Tendera, P. Widimsky, J. L. Zamorano, S. Erdine, W. Kiowski, E. Agabiti-Rosei, E. Ambrosioni, L. H. Lindholm, M. Viigimaa, S. Adamopoulos, E. Agabiti-Rosei, E. Ambrosioni, V. Bertomeu, D. Clement, S. Erdine, C. Farsang, D. Gaita, G. Lip, J. M. Mallion, A. J. Manolis, P. M. Nilsson, E. O'Brien, P. Ponikowski, J. Redon, F. Ruschitzka, J. Tamargo, P. van Zwieten, B. Waeber and B. Williams, "Management of Arterial Hypertension of the European Society of Hypertension; European Society of Cardiology. 2007 Guidelines for the Management of Arterial Hypertension: The Task Force for the Management of Arterial Hypertension of the European Society of Hypertension (ESH) and of the European Society of Cardiology (ESC)," Journal of Hypertension, Vol. 25, 2007, pp. 1105-1187. doi:10.1097/HJH.0b013e3281fc975a

[32] M. van den Heuvel, W. W. Batenburg, S. Jainandunsing, I. M. Garrelds, J. M. G. van Gool, R. A. Feelders, A. H. van den Meiracker and A. H. Jan Danser, "Urinary Renin, but Not Angiotensinogen or Aldosterone, Reflects the Renal Renin-Angiotensin-Aldosterone System Activity and the Efficacy of Renin-Angiotensin-Aldosterone System Blockade in the Kidney," Journal of Hypertension, Vol. 29, 2011, pp. 2147-2155. doi:10.1097/HJH.0b013e32834bbcbf

[33] T. Ogihara, K. Kikuchi, H. Matsuoka, T. Fujita, J. Higaki, M. Horiuchi, Y. Imai, T. Imaizumi, S. Ito, H. Iwao, K. Kario, Y. Kawano, S. Kim-Mitsuyama, G. Kimura, H. Matsubara, H. Matsuura, M. Naruse, I. Saito, K. Shimada, K. Shimamoto, H. Suzuki, S. Takishita, N. Tanahashi, T. Tsuchihashi, M. Uchiyama, S. Ueda, H. Ueshima, S. Umemura, T. Ishimitsu and H. Rakugi, "The Japanese Society of Hypertension Guidelines for the Management of Hypertension (JSH 2009)," Hypertension Research, Vol. 32, 2009, pp. 11-23. doi:10.1038/hr.2009.34

[34] A. V. Chobanian, G. L. Bakris, H. R. Black, W. C. Cushman, L. A. Green, J. L. Izzo Jr., D. W. Jones, B. J. Materson, S. Oparil, J. T. Wright Jr. and E. J. Roccella, "National Heart, Lung, and Blood Institute Joint National Committee on Prevention, Detection, Evaluation, and Treatment of High Blood Pressure; National High Blood Pressure Education Program Coordinating Committee. The Seventh Report of the Joint National Committee on Prevention, Detection, Evaluation, and Treatment of High Blood Pressure: The JNC 7 Report," JAMA, Vol. 289, No. 19, 2003, pp. 2560-2572. doi:10.1001/jama.289.19.2560 